\title{
Stability of Fourier Solutions of Nonlinear Stochastic Heat Equations in 1D
} Hazaimeh $\mathbf{M H}^{*}$

Department of Mathematics, Zayed University, Dubai, UAE

\begin{abstract}
The main focus of this article is studying the stability of solutions of nonlinear stochastic heat equation and give conclusions in two cases: stability in probability and almost sure exponential stability. The main tool is the study of related Lyapunov-type functionals. The analysis is carried out by a natural $\mathrm{N}$-dimensional truncation in isometric Hilbert spaces and uniform estimation of moments with respect to $N$.
\end{abstract}

Nonlinear stochastic heat equation, additive space-time noise, Lyapunov functional, Fourier solution, finitedimensional approximations, moments, stability.

Keywords: Nonlinear stochastic heat equation; Additive spacetime noise; Lyapunov functional; Fourier solution; Finite-dimensional approximations; Moments; Stability

\section{Introduction}

In this article we study the stability of solutions of semi-linear stochastic heat equations

$$
u_{t}=\sigma^{2} \Delta u+A(u)+B(u) \frac{d W}{d t}
$$

with cubic nonlinearities $A(u)$ in one dimensions in terms of all systems parameters, i.e., with non-global Lipschitz continuous nonlinearities. Our study focusses on stability of analytic solution $u=u(x, t)$ under the geometric condition

$$
\sigma^{2} \frac{\pi^{2}}{l^{2}}-a_{1}=\frac{\sigma^{2} \pi^{2} l^{2}-a_{1} l^{2}}{l^{2}}:=\gamma>0,
$$

where $0 \leq x \leq 1$ such that $\mathrm{D}=[0, l]$.

Many authors have treated stochastic heat equations (e.g. [1,2]), semi-linear stochastic heat equations (e.g. [1-3]) or nonlinear stochastic evolution equations (e.g. $[4,5])$. Also, some authors study the stability of stochastic heat equations like Fournier and Printems [6] study the stability of the mild solution. Walsh reats the stochastic heat equations in one dimension. Chow [1] studies that the null solution of the stochastic heat equation is stable in probability by using the definition. Recall that:

$$
\mathbb{L}^{2}(\mathbb{D})=\left\{f:\left.\mathbb{D} \rightarrow \mathbb{R}\left|\int_{\mathbb{D}}\right| f(x)\right|^{2} d \mu(x)<\infty\right\},
$$

Where $\mu$ is the Lebesgue measure in one dimensions. The paper is organized as follows. Section 2 states that the strong Fourier solution of equation (1) is proved. We write the solution using the finite-dimensional truncated system verifies properties of finitedimensional Lyapunov functional. Section 3 discusses the stability of the strong solution of equation (1) is stable in probability and almost sure exponential stability. Eventually, Section 4 summarizes the most important conclusions on the well-posedness and behaviour of the original infinite-dimensional system (1).

\section{Truncated Fourier Series Solution and Finite- dimensional Lyapunov Functional}

Consider the stochastic nonlinear heat equation with additive noise

$u_{t}=\sigma^{2} u_{x x}+\left(a_{1}-a_{2}\|u\|_{\mathbb{L}^{2}(\mathbb{D})}^{2}\right) u+b \frac{d W}{d t}$

with the initial condition $u(x, 0)=f(x)$ with $f \in \mathrm{L}^{2}(\mathrm{D})$ (initial position) and $W(x, t)=\sum_{n=1}^{\infty} \alpha_{n} W_{n}(t) e_{n}(x)$ and $e_{n}=\sqrt{\frac{2}{l}} \sin \left(\frac{n \pi x}{l}\right)$ driven by i.i.d. standard Wiener processes $W_{n}$ with $\mathrm{E}\left[W_{n}(t)\right]^{2}=0, \mathrm{E}\left[W_{n}(t)\right]^{2}=t$. The solution of equation (1) in terms of Fourier series is proved by Schurz [3] and given by

$$
u(x, t)=\sum_{n=1}^{\infty} c_{n}(t) e_{n}(x)
$$

\section{Theorem 1}

Assume that $\sum_{n, m=1}^{\infty} \alpha_{n}^{2}<\infty, \quad \forall u \in \mathbb{L}^{2}(\mathbb{D}) \cap C^{1}\left(\mathbb{D} \times \mathbb{R}_{+}^{1}\right)$ with $u_{x} \in$ $\mathrm{L}^{2}(\mathrm{D})$ and $W(x, t)=\sum_{n, m=1}^{\infty} \alpha_{n} W_{n}(t) e_{n}(x)$, then for all $t \geq 0, x \in \mathrm{D}=\left(0, l_{x}\right)$, the Fourier-series solutions (2) have Fourier coefficients $c_{n}$ satisfying (a.s.)

$$
\frac{d}{d t} c_{n}(t)=\left[-\sigma^{2} \frac{\pi^{2} n^{2}}{l^{2}}+a_{1}-a_{2} \sum_{k=1}^{\infty}\left(c_{k}\right)^{2}\right] c_{n}+b \alpha_{n} \frac{d W_{n}}{d t} .
$$

Proof. See Schurz [3].

We need to truncate the infinite series (2) for practical computations. So, we have to consider finite-dimensional truncations of the form

$$
u_{N}(x, t)=\sum_{n=1}^{N} c_{n} e_{n}
$$

with Fourier coefficients $c_{n}$ satisfying the naturally truncated system of stochastic differential equations (SDEs).

$$
\frac{d}{d t} c_{n}(t)=\left[-\sigma^{2} \frac{\pi^{2} n^{2}}{l^{2}}+a_{1}-a_{2} \sum_{k=1}^{\infty}\left(c_{k}\right)^{2}\right] c_{n}+b \alpha_{n} \frac{d W_{n}}{d t} .
$$

where $\lambda_{n}=\left(\frac{n \pi}{l}\right)^{2}$

Assume that $\sigma^{2} \frac{\pi^{2}}{l^{2}}>a_{1}$. Define the Lyapunov functional $V_{N}$ as follows

*Corresponding author: Hazaimeh $\mathrm{MH}$, Department of Mathematics, University College, Zayed University, P.O.Box 19282, Dubai, UAE, Tel: +971 44021111 E-mail: haziem67@gmail.com

Received September 22, 2016; Accepted October 05, 2016; Published October 15,2016

Citation: Hazaimeh MH (2016) Stability of Fourier Solutions of Nonlinear Stochastic Heat Equations in 1D. J Appl Computat Math 5: 323. doi: 10.4172/21689679.1000323

Copyright: ( 2016 Hazaimeh MH. This is an open-access article distributed under the terms of the Creative Commons Attribution License, which permits unrestricted use, distribution, and reproduction in any medium, provided the original author and source are credited. 


$$
\begin{aligned}
& V_{N}(c)=V_{N}\left(\left(c_{n}\right)_{n=1, \ldots, N}\right):=\sum_{n=1}^{N}\left(\sigma^{2} \lambda_{n}-a_{1}\right)\left(c_{n}\right)^{2}+\frac{a_{2}}{2}\left(\sum_{n=1}^{N}\left(c_{n}\right)^{2}\right)^{2} \\
& \text { for } N \in \mathrm{N} .
\end{aligned}
$$

This functional is a modification of a functional appeared in Schurz [7]. It is clear that this function is of Lyapunov-type because it is nonnegative and smooth as long as $a_{2} \geq 0$, radially unbounded if additionally $\sigma^{2} \pi^{2}>a_{1} l^{2}$. Equipped with Euclidean norm

$$
|c|_{l_{N}^{2}}=\sqrt{\sum_{n=1}^{N} c_{n}^{2}}
$$

\section{Lemma 2}

Consider the Lyapunov functional defined in equation (6), and let $\sigma^{2} \frac{\pi^{2}}{l^{2}}-a_{1}=\frac{\sigma^{2} \pi^{2}-a_{1} l^{2}}{l^{2}}=: \gamma>0$.

Then $\forall u \in \mathrm{L}^{2}(\mathrm{D})$ :

$V_{N}(u) \geq \gamma\|u\|_{\mathbb{L}^{2}(\mathbb{D})}^{2}$

Proof. See [7].

\section{Lemma 3}

Assume that $a_{2} \geq 0$. Then, $\forall N \in \mathrm{N}$, the functional $V_{N}$ is

(a) nonnegative and positive semi-definite if $\sigma^{2} \pi^{2}>a_{1} l^{2}$ or $a_{2} \geq 0$.

(b) positive-definite if $\sigma^{2} \pi^{2}>a_{1} l^{2}$, and

(c) satisfies the condition of radial unboundedness

$\lim _{|c| l_{N}^{2} \rightarrow+\infty} V_{N}(c)=+\infty$, if $\left[\sigma^{2} \pi^{2}-a_{1} l^{2}\right]_{+}+a_{2}>0$.

Proof. See [7]

\section{Stability of Fourier Solutions}

Recall equation (5) governed by

$$
\begin{aligned}
& \frac{d}{d t} c_{n}(t)=\left[-\sigma^{2} \frac{\pi^{2} n^{2}}{l^{2}}+a_{1}-a_{2} \sum_{k=1}^{\infty}\left(c_{k}\right)^{2}\right] c_{n}+b \alpha_{n} \frac{d W_{n}}{d t} \\
& =\left[-\sigma^{2} \lambda_{n}+a_{1}-a_{2}\left\|u_{N}\right\|^{2}\right] c_{n}+b \alpha_{n} \frac{d W_{n}}{d t} .
\end{aligned}
$$

To simplify, let

$$
\begin{aligned}
& f\left(u_{N}\right)=-\sigma^{2} \lambda_{n}+a_{1}-a_{2}\left\|u_{N}\right\|^{2} \\
& \text { and } \\
& g\left(u_{N}\right)=b \alpha_{n}
\end{aligned}
$$

Definition: The trivial solution of system (8) (in terms of norm $\left.\|u\|_{\mathbb{L}^{2}(\mathbb{D})}\right)$ is said to be stochastically stable or stable in probability, if for $0<$ $\varepsilon<1$ and $r>0, \exists a \delta=\delta(\varepsilon, r)$ such that, $\forall t \geq \delta$, we have

$\mathbb{P}\left\{\|u(t)\|_{\mathbb{L}^{2}(\mathbb{D})}<r\right\} \geq 1-\varepsilon$.

whenever $\delta>0$.

\section{Lemma 4}

If $\exists$ a positive-definite function $V \in \mathrm{C}^{2,1}\left(\mathrm{R}^{d} \times[0, \infty), \mathrm{R}_{+}\right)$such that $\mathrm{L} V(x, t) \leq 0$ and $\forall(x, t) \in \mathrm{R}^{d} \times[0 . \infty)$, then the trivial solution of the equation. $d X(t)=f(x(t), t) d t+g(x(t), t) d w(t)$

is stochastically stable.

Proof. See Arnold [8].

\section{Theorem 5}

Let $V(u(t))=\sigma^{2}\|\nabla u\|_{\mathbb{L}^{2}(\mathbb{D})}^{2}-a_{1}\|u\|_{\mathbb{L}^{2}(\mathbb{D})}^{2}+\frac{a_{2}}{2}\|u\|_{\mathbb{L}^{2}(\mathbb{D})}^{4}$

If $\left(1-a_{2} \sum_{n=1}^{N} c_{n}^{2}\right) \sum_{n=1}^{N} c_{n}>0$, then the trivial solution of equation (8) is stochastically stable i.e., stable in probability.

Proof. From Lemma 3, we know that $\mathrm{V}_{N}(u(t))$ is positive-definite if $\forall n \mathrm{~N}, \sigma^{2} \lambda_{\mathrm{n}}-a_{1}>0$. Define the linear operator $\mathrm{L}$ as in Schurz [3]

$\mathcal{L}=\sum_{n=1}^{N}\left[-\sigma^{2} \frac{\pi^{2} n^{2}}{l^{2}}+a_{1}-a_{2} \sum_{k=1}^{N}\left(c_{k}\right)^{2}\right] c_{n} \frac{\partial}{\partial c_{n}}+\frac{b^{2}}{2} \sum_{n=1}^{N} \alpha_{n}^{2} \frac{\partial^{2}}{\partial c_{n}^{2}}$.

The first and second partial derivative of $V_{N}(t)$ with respect to $c_{n}$ are $\frac{\partial V_{N}}{\partial c_{n}}=2 \sum_{n=1}^{N}\left[\left(\sigma^{2} \lambda_{n}-a_{1}\right)+a_{2}\left(\sum_{n=1}^{N} c_{n}^{2}\right)\right] c_{n}$

and

$\frac{\partial^{2} V_{N}}{\partial c_{n}^{2}}=2 \sum_{n=1}^{N}\left(\sigma^{2} \lambda_{n}-a_{1}\right) c_{n}+4 a_{2}\left(\sum_{n=1}^{N} c_{n}\right)^{2}+2 a_{2} \sum_{n=1}^{N} c_{n}^{2}$.

Then

$$
\begin{aligned}
& \mathcal{L} V_{N}\left(c_{n}(t)\right)=-2\left(\sum_{n=1}^{N}\left(\sigma^{2} \lambda_{n}-a_{1}\right) c_{n}\right)^{2}-2 a_{2} \sum_{n=1}^{N}\left(\sigma^{2} \lambda_{n}-a_{1}\right)\left(\sum_{n=1}^{N} c_{n}^{2}\right)^{2} \\
& -2 a_{2} \sum_{n=1}^{N} c_{n}^{2}\left(1-a_{2} \sum_{n=1}^{N} c_{n}^{2} \sum_{n=1}^{N}\left[\left(\sigma^{2} \lambda_{n}-a_{1}\right)\right] c_{n} .\right.
\end{aligned}
$$

But by our assumption that

$$
\left(1-a_{2} \sum_{n=1}^{N} c_{n}^{2}\right) \sum_{n=1}^{N} c_{n}>0
$$

Then thus

$\operatorname{LV}_{N}\left(c_{n}(t)\right) \leq 0$.

So by Lemma 4, applied to truncation of (8), the trivial solution of system (8) is stochastically stable.

\section{Corollary 6}

Let $p \geq 2$ and let $V$ be as above. Imposing the same assumptions as in Theorem 5 with $N \rightarrow+\infty$, then we have $\forall 0 \leq t \leq T$,

$$
\mathbb{E}\|u(t)\|_{\mathbb{L}^{2}(\mathbb{D})}^{p} \leq \frac{1}{\min (1, \gamma)} \mathbb{E} V^{\frac{p}{2}}(u(0)) .
$$

Proof. We know, from the definition of $V(u)$, and Lemma 2 that $\|u\|_{\mathbb{L}^{2}(\mathbb{D})}^{2} \leq \frac{V(u)}{\gamma}$. it is easy to show that

$$
\mathbb{E}\|u(t)\|_{\mathbb{L}^{2}(\mathbb{D})}^{p} \leq \frac{1}{\min (1, \gamma)} \mathbb{E} V^{\frac{p}{2}}(u(0)) .
$$

\section{Corollary 7}

$\forall p \geq 2$ and $\forall 0 \leq t \leq T$, with $\sigma^{2} \lambda_{1}-a_{1}>0$, we have $\forall 0 \leq t \leq T$.

1) If $a_{2} \geq 0$, then

$\mathbb{E}\|u(t)\|_{\mathbb{L}^{2}(\mathbb{D})}^{p} \leq \frac{\mathbb{E} V^{\frac{p}{2}}(u(0))}{\left[\sigma^{2} \lambda_{1}-a_{1}\right]^{\frac{p}{2}}}$.

2) If $a_{2}>0$, then 


$$
\mathbb{E}\|u(t)\|_{\mathbb{L}(\mathbb{D})} \leq\left(-\frac{p}{4}\right)^{\frac{p}{4}} V^{\frac{p}{4}}(u(0)) .
$$

Proof. 1) Note that we have $\left(\sigma^{2} \lambda_{1}-a_{1}\right)\|u(., t)\|_{\mathbb{L}^{2}(\mathbb{D})}^{2} \leq V_{N}(u(t))$. Since $\lambda_{n}$ is increasing in $n$,

$$
\left[\sigma^{2} \lambda_{1}-a_{1}\right]\|u(t)\|_{\mathbb{L}^{2}(\mathbb{D})}^{2} \leq V_{N}(u(t))
$$

So,

$$
\|u(t)\|_{\mathbb{L}^{2}(\mathbb{D})}^{2} \leq \frac{V_{N}(u(t))}{\sigma^{2} \lambda_{1}-a_{1}}
$$

Pull over expectation, then

$$
\mathbb{E}\|u(t)\|_{\mathbb{L}^{2}(\mathbb{D})}^{2} \leq \frac{\mathbb{E} V(u(t))}{\sigma^{2} \lambda_{1}-a_{1}} .
$$

By using Corollary 6, we have

$$
\mathbb{E}\|u(t)\|_{\mathbb{L}^{2}(\mathbb{D})}^{2} \leq \frac{\mathbb{E} V(u(0))}{\sigma^{2} \lambda_{1}-a_{1}} .
$$
so

2) From the definition of $V(u(t))$, it is clear that $\frac{a_{2}}{2}\|u(t)\|_{\mathbb{L}^{2}(\mathbb{D})}^{4} \leq V(u(t))$,

$$
\left(\frac{a_{2}}{2}\right)\|u(t)\|_{\mathbb{L}^{2}(\mathbb{D})}^{4} \leq V_{N}(u(0)) .
$$

Now, take the expectation to both sides, and we get

$$
\begin{aligned}
& \left(\frac{a_{2}}{2}\right) \mathbb{E}\|u(t)\|_{\mathbb{L}^{2}(\mathbb{D})}^{4} \leq \mathbb{E} V_{N}(u(0)), \\
& \text { i.e., } \forall 0 \leq t \leq T, \\
& \mathbb{E}\|u(t)\|_{\mathbb{L}^{2}(\mathbb{D})}^{2} \leq\left(\frac{2}{a_{2}}\right)^{\frac{1}{2}} \mathbb{E} V^{\frac{1}{2}}(u(0)) .
\end{aligned}
$$

Remark: The corollary 7 means that $\forall t \geq 0$ :

$$
\mathbb{E}\|u(t)\|_{\mathbb{L}^{2}(\mathbb{D})}^{2} \leq \min \left\{\frac{\mathbb{E} V_{N}(u(0))}{\left[\sigma^{2} \lambda_{1}-a_{1}\right]},\left(\frac{2}{a_{2}}\right)^{\frac{1}{2}} \mathbb{E} V^{\frac{1}{2}}(u(0))\right\} .
$$

Definition: The trivial solution of system (8) is said to be a.s. exponentially stable if

$$
\theta\left(u_{N}\right):=\limsup _{t \rightarrow \infty} \frac{1}{t} \log \|u(t)\|_{\mathbb{L}^{2}(\mathbb{D})}<0 \quad \text { (a.s.) }
$$

$\forall u(0) \in \mathrm{D}$. The quantity of the left hand side of (12) is called the sample top Lyapunov exponent of $u$.

\section{Lemma 8}

Let $v(t)$ be a nonnegative integrable function such that [9]

$$
v(t) \leq C+A \int_{0}^{t} v(s) d s, \quad 0 \leq t \leq T
$$

for some constants $\mathrm{C}$, $\mathrm{A}$. Then $C \geq 0$ and

$\mathrm{v}(\mathrm{t}) \leq C \exp (A t), 0 \leq \mathrm{t} \leq \mathrm{T}$

\section{Theorem 9}

Let $V(u(t))$ as in Theorem 5. If $\left(1-a_{2} \sum_{n=1}^{N} c_{n}^{2}\right) \sum_{n=1}^{N} c_{n}>0$, then the norm of the trivial solution of $\mathrm{N}$-dimensional system (8) is a.s. exponentially stable with sample top Lyapunov exponent

$$
\theta\left(u_{N}\right) \leq 0 \text {. }
$$

Proof. Return to the analysis of finite $\mathrm{N}$-dimensional equation (5). Recall that

$$
\begin{aligned}
& V\left(u_{N}(t)\right)=\sigma^{2}\left\|\nabla u_{N}\right\|_{\mathbb{L}^{2}(\mathbb{D})}^{2}-a_{1}\left\|u_{N}\right\|_{\mathbb{L}^{2}(\mathbb{D})}^{2}+\frac{a_{2}}{2}\left\|u_{N}\right\|_{\mathbb{L}^{2}(\mathbb{D})}^{4} \\
& =\sum_{n=1}^{N}\left[\sigma^{2} \lambda_{n}-a_{1}\right]\left(c_{n}(t)\right)^{2}+\frac{a_{2}}{2}\left(\sum_{n=1}^{N}\left(c_{n}(t)\right)^{2}\right)^{2} \\
& \text { where } V\left(u_{N}\right)=V_{N}(c) \text { and from Theorem } 5 \text { we know that } \\
& \mathcal{L} V_{N}\left(c_{n}(t)\right)=-2\left(\sum_{n=1}^{N}\left(\sigma^{2} \lambda_{n}-a_{1}\right) c_{n}\right)^{2}-2 a_{2} \sum_{n=1}^{N}\left(\sigma^{2} \lambda_{n}-a_{1}\right)\left(\sum_{n=1}^{N} c_{n}^{2}\right)^{2}
\end{aligned}
$$

$$
-2 a_{2} \sum_{n=1}^{N} c_{n}^{2}\left(1-a_{2} \sum_{n=1}^{N} c_{n}^{2}\right) \sum_{n=1}^{N}\left[\left(\sigma^{2} \lambda_{n}-a_{1}\right)\right] c_{n} .
$$

But by our assumption that

$$
\begin{aligned}
& \left(1-a_{2} \sum_{n=1}^{N} c_{n}^{2}\right) \sum_{n=1}^{N} c_{n}>0, \\
& \text { so } \\
& \mathcal{L} V_{N}\left(c_{n}(t)\right) \leq-k,
\end{aligned}
$$

where $k \geq 0$.

Using Dynkin's formula, we find that [10-16]

$$
\begin{aligned}
& \mathbb{E} V_{N}(c(t))=\mathbb{E} V_{N}(c(0))+\mathbb{E} \int_{0}^{t} \mathcal{L} V_{N}\left(c_{n}(s)\right) d s \\
& \leq \mathbb{E} V_{N}(c, v)(0)-k t
\end{aligned}
$$

so

$$
\mathbb{E}\left\|u_{N}(t)\right\|_{\mathbb{L}^{2}(\mathbb{D})}^{2} \leq \mathbb{E} V_{N}(c(t)) \leq \mathbb{E} V_{N}(c(0))-k t
$$

using extended Gronwall lemma, Lemma 8, gives us

$\mathbb{E}\left\|u_{N}(t)\right\|_{\mathbb{L}^{2}(\mathbb{E})}^{2} \leq \mathbb{E} V_{N}(c(0)) e^{-k t}$

hence

$\log \mathbb{E}\left\|u_{N}(t)\right\|_{\mathbb{L}^{2}(\mathbb{D})}^{2} \leq \log \mathbb{E} V_{N}(c(0))-k t$

thus

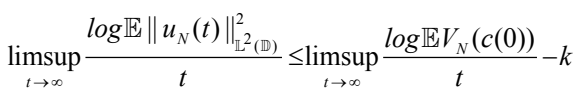

$$
\begin{aligned}
& \leq b_{2}^{2}\|\alpha\|_{1_{4 N \times N}^{2}}^{2}-2 \kappa \leq-k \text {. }
\end{aligned}
$$

If $\left(1-a_{2} \sum_{n=1}^{N} c_{n}^{2}\right) \sum_{n=1}^{N} c_{n}>0$ then the left side of identity (12) is negative and the trivial solution of the velocity $v$ of $\mathrm{N}$-dimensional system (8) is a.s. exponential stable.

Finally, we observe that all the previous estimates are uniformly bounded as $\mathrm{N} \rightarrow \infty$. Hence, we arrive at

$$
\underset{t \rightarrow \infty}{\limsup } \frac{\log \mathbb{E}\|u(t)\|_{\mathbb{L}^{2}(\mathbb{D})}^{2}}{t}<0 .
$$

\section{Corollary 10}

Let $V(u(t))$ as in Theorem 5. If $\left(1-a_{2} \sum_{n=1}^{N} c_{n}^{2}\right)$, then the norm of the $v$-component of the trivial solution of infinite-dimensional system (1) is a.s. exponentially stable with sample top Lyapunov exponent

$$
\theta\left(v_{N}\right) \leq-k<0 .
$$

Proof. Return to the proof of previous Theorem 9 and take the limit $N$ to $+\infty$ after the estimation process (16) in the sample Lyapunov exponent $\theta\left(v_{N}\right)$.

\section{Conclusion}

By analyzing appropriate $N$-dimensional truncations of the original semi-linear heat equations (1), we can verify the asymptotic stability of random Fourier series solutions with strongly unique, Markovian, continuous time Fourier coefficients under the presence of cubic nonlinearities. For this purpose, we introduced and studied an appropriate Lyapunov. The analysis is basicly relying on the fact that all estimations of moments of Lyapunov functional are made independent of dimensions $N$ of their finite-dimensional truncations. Thus, the techniques of our proof are finite-dimensional in character, however 
Citation: Hazaimeh MH (2016) Stability of Fourier Solutions of Nonlinear Stochastic Heat Equations in 1D. J Appl Computat Math 5: 323. doi: 10.4172/2168-9679.1000323

Page 4 of 4

the conclusions can be drawn to the original infinite-dimensional semilinear equation.

\section{References}

1. Chow PL (2007) Stochastic Partial Differential Equations. Chapman and Hall/ CRC, Boca Raton.

2. Da Prato G, Zabzcyk J (1992) Stochastic Equations in Infinite Dimensions, Cambridge University Press, Cambridge, London.

3. Schurz H (2010) Nonlinear stochastic heat equations with cubic nonlinearity and additive Q-reglar noise in R1. Electronic Journal of Differential Equations Conf 19: 221-233.

4. Grecksch W, Tudor C (1995) Stochastic Evolutions: A Hilbert Space Approach Akademie-Verlag, Berlin.

5. Schurz $H$ (2007) Existence and uniqueness of solutions of semilinear stochastic infinite-dimensional differential systems with $\mathrm{H}$-Regular noise. J Math Anal Appl 332: 334-345.

6. Fournier N, Printems J (2011) Stability of the Stochastic Heat Equation in L1 $([0,1])$. Elect Comm in Probab 16: 337-352.

7. Schurz H, Hazaimeh HM (2014) Existence, Uniqueness, and stability of stochastic wave equation with cubic nonlinearities in two dimensions. Journal of Mathematical Analysis and Applications 148: 775-795.
8. Arnold L (1974) Stochastic Differential Equations: Theory and Applicants. John Wiley and Sons, Inc., New York.

9. Ksendal BØ (2003) Stochastic Differential Equations: An Introduction With applications, Springer-Verlag, Berlin.

10. Dalang R, Frangos NE (1998) The stochastic wave equation in two spatia dimensions. Ann Probab 26: 187-212.

11. Friedman A (1975) Stochastic Differential Equations and Applications. Dover Publications Inc. Mineola, New York.

12. Gard TC (1988) Introduction to Stochastic Differential Equations. Marce Dekker, Basel.

13. Karatzas I, SchreveSE (1991) Brownian Motion and Stochastic Calculus. Springer-Verlag, New York.

14. Millet A, Sanz-Sole M (1999) A stochastic wave equation in two space dimension: Smootheness of the law. Ann Probab 27: 803-844.

15. Shiryaev (1996) Probability (2ndedn) Springer-Verlag, Berlin.

16. Walsh JB (1986) An introduction to stochastic partial differential equations École d'été de probabilités de Saint-Flour, XIV-1984, Springer-Verlag, Berlin pp: 265-439. 